\title{
Comorbid Illness Is an Important Determinant of Health-Related Quality of Life in Patients With Chronic Hepatitis C
}

Khozema B. Hussain, M.D., Robert J. Fontana, M.D., Cheryl A. Moyer, M.S., Grace L. Su, M.D., Naomi Sneed-Pee, B.S., and Anna S. F. Lok, M.D.

Division of Gastroenterology and Consortium for Health Outcomes Innovations and Cost-effectiveness Studies (CHOICES), University of Michigan Health System, Ann Arbor, Michigan

OBJECTIVES: Chronic hepatitis C (CHC) patients selected for entry into treatment trials have been reported to have impaired health-related quality of life (HRQOL). However, these trials have an inherent selection bias, and HRQOL in CHC patients may have been underestimated because of the exclusion of patients with comorbid illness. The aim of this study was to assess HRQOL in an unselected group of $\mathrm{CHC}$ patients and to identify factors associated with impairment in HRQOL.

METHODS: A total of 220 consecutive eligible CHC patients were enrolled from a hepatology clinic. HRQOL was assessed by the short form 36 (SF-36) and comorbid illnesses were assessed by an interview.

RESULTS: CHC patients had significantly lower SF-36 scores in all subscales and in the summary scales when compared to those of the healthy general population in the United States $(p<0.001)$. Compared to CHC patients entering treatment trials, our patients had lower SF-36 scores on five subscales $(p<0.001)$. The presence of comorbid illness was the most important predictor of HRQOL in CHC patients. However, CHC alone resulted in significantly lower SF-36 scores in all subscales and summary scales $(p \leq 0.003)$ compared to those of the healthy U.S. population. There was no correlation between SF-36 scores and history of i.v. drug use or dependence, alcohol dependence, and serum aminotransferase levels.

CONCLUSIONS: We conclude that unselected CHC patients presenting for medical evaluation have a reduced HRQOL, which is lower than that reported for $\mathrm{CHC}$ patients entering treatment trials. $\mathrm{CHC}$ alone is associated with significant impairment in HRQOL, but the presence of comorbid illness leads to further diminution in HRQOL. (Am J Gastroenterol 2001;96:2737-2744. (C) 2001 by Am. Coll. of Gastroenterology)

\section{INTRODUCTION}

Approximately $1-2 \%$ of the U.S. population, or an estimated 4 million Americans, are chronically infected with hepatitis C (1). Although most chronic hepatitis C (CHC) patients have few or no symptoms during the early stages of their illness, several studies have reported impairment in health-related quality of life (HRQOL) compared to healthy individuals (2-7). Most studies have used an instrument based on the short-form 36 (2-6) developed by the Medical Outcomes Trust $(8,9)$. All but one of the published studies on HRQOL in CHC patients have focused on patients selected for enrollment into treatment trials $(2,4-6)$. In one study, HRQOL was assessed in 642 treatment-naive CHC patients who participated in a multicenter treatment trial. Before treatment, the $\mathrm{CHC}$ patients had markedly impaired quality of life compared to that of the healthy U.S. population (2). During posttreatment follow-up, significant improvement in HRQOL was observed among the sustained responders but not in the nonresponders or relapsers, supporting a causal relation between viral replication and impairment in HRQOL. However, sustained responders continued to have impaired HRQOL compared to healthy individuals, suggesting that residual liver disease or factors besides hepatitis $\mathrm{C}$ may be responsible for the persistent impairment in HRQOL in these patients. These studies clearly demonstrate a consistent and marked reduction in HRQOL among CHC patients. However, the magnitude of reduction in HRQOL associated with $\mathrm{CHC}$ may have been underestimated because of selection bias, because patients with psychiatric illness, polysubstance abuse or multiple comorbid medical illnesses are usually excluded from treatment trials. Serum aminotransferase (ALT) level (2-6), presence of cirrhosis on liver biopsy $(3,6,10)$, and viral factors $(2,6)$ have not been shown to correlate with HRQOL in $\mathrm{CHC}$ patients.

Among the published studies on HRQOL in CHC patients, only one was conducted outside the context of a treatment trial (3). This study found that CHC patients had not only lower HRQOL scores than those of healthy controls, but also lower scores than those of chronic hepatitis B patients $(\mathrm{N}=30)$ with similar severity of liver disease. We hypothesize that unselected $\mathrm{CHC}$ patients have more marked impairment in HRQOL than that reported in patients 
enrolled in clinical treatment trials. We further hypothesize that although CHC itself may result in impaired HRQOL, extrahepatic factors such as history of drug or alcohol abuse, as well as concomitant medical or psychiatric illness may be important determinants of HRQOL in CHC patients (11). The aims of our study were to assess HRQOL in consecutive CHC patients attending our General Hepatology Clinic and to identify factors that correlate with HRQOL in CHC patients.

\section{MATERIALS AND METHODS}

\section{Study Population}

Consecutive eligible CHC patients attending the General Hepatology Clinic at the University of Michigan Hospital during the 7-month period between October, 1999, and May, 2000, were invited to participate in the study. Inclusion criteria were detectable hepatitis $\mathrm{C}$ antibody or hepatitis $\mathrm{C}$ virus RNA (HCV RNA), with or without elevation in serum ALT level. Both men and women, of all races, between the ages of 15 and $70 \mathrm{yr}$ were included. Patients who were receiving or had received treatment with interferon alone or in combination with ribavirin during the past 3 months were excluded, as were sustained responders to previous therapy. Patients with decompensated liver disease, coinfection with hepatitis B or HIV, other causes of liver disease, malignancies in the past $1 \mathrm{yr}$, life-threatening medical problems, or marrow/organ transplantations were also excluded. The study was approved by the institutional review board at the University of Michigan Hospital, and written informed consent was obtained from each patient.

Potentially eligible participants were identified $2 \mathrm{wk}$ before their clinic appointment by screening the physicians' schedules, referral letters, and computerized medical records. A letter of invitation was mailed to eligible patients together with a copy of the consent form. The patients received up to two follow-up phone calls 1 wk later to explain the study and to inquire about their interest in participation. Interested individuals were scheduled to complete a confidential survey in a private area before their physician appointment. Medical records and laboratory test results were reviewed after the clinic visit to confirm participant eligibility.

\section{Data Collection}

A 67-item, self-administered survey with the SF-36 embedded within it was used to collect data on HRQOL, demographics, risk factors for $\mathrm{HCV}$ infection, patient knowledge about transmission and prognosis of $\mathrm{CHC}$, and alcohol and drug use history. The SF-36 is a self-administered generic instrument that assesses a patient's QOL along eight subscales and two summary scales. The subscales include physical functioning (PF), role-physical (RP), role-emotional (RE), vitality (VT), mental health (MH), social functioning (SF), general health (GH), and bodily pain (BP). The sum- mary scales consist of physical health (PCS) and mental health (MCS). The SF-36 subscale and summary scores range from 0 to 100 , with higher scores reflecting better HRQOL.

Patients who reported a history of i.v. drug use or a history of regular alcohol consumption (defined as two drinks daily for $>3$ months) were asked if they ever considered themselves to be dependent on drugs or alcohol. Drug and alcohol dependence was further assessed by inquiring about the duration of use and the need for counseling or rehabilitation. History of current comorbid medical or psychiatric illness was obtained during a brief interview and corroborated with physicians' notes and medication lists. Completion of the survey and medical interview required an average of $45 \mathrm{~min}$. Reports of liver biopsies performed within the last $2 \mathrm{yr}$ were reviewed for the presence or absence of cirrhosis. Serum ALT levels within 3 months of the study visit were recorded.

\section{Data Analyses}

Descriptive statistics were calculated and reported as means \pm SD unless indicated otherwise. SF-36 scores were calculated using SAS software (SAS, Cary, NC). Statistical analysis was performed using SPSS for Windows 1997 (SPSS, Chicago, IL). Normative population data for SF-36 scores were obtained through the Medical Outcomes Trust $(12,13)$. These data were based on a sample $(\mathrm{N}=2474)$ of noninstitutionalized members of the general U.S. population, aged 18-94 yr, gathered from October through December, 1990. SF-36 subscale and summary scores were compared to healthy and general U.S. population controls using analysis of variance. Univariate analysis of associations among clinical variables of interest and the 10 subscales and summary scores of the SF-36 were determined using correlations, $\chi^{2}$, analysis of variance, and KruskalWallis tests. Because multiple comparisons were conducted, a $p$ value of $<0.01$ was considered statistically significant.

\section{RESULTS}

\section{Characteristics of the Study Population}

A total of 674 patients with CHC attended the General Hepatology Clinic at the University of Michigan Hospital during the 7-month study period. Of these, 268 patients were ineligible for the study: 136 patients had decompensated liver disease; 68 were either currently receiving or had received antiviral therapy within the past 3 months; 12 were sustained responders to previous antiviral therapy; and 52 met other exclusion criteria.

Of the 406 CHC eligible patients, 220 (54\%) patients were enrolled in the study. The remaining 186 patients were unable to participate, primarily because of time constraints during their clinic appointments.

A total of 139 (63\%) participants were men (Table 1). The mean age was $46.6 \pm 7.9 \mathrm{yr}$. In all, $81 \%$ were Cauca- 
Table 1. Characteristics of the Study Population

\begin{tabular}{|c|c|c|c|}
\hline & CHC Study Group & Nonparticipants & $p$ \\
\hline No. of patients & 220 & 186 & \\
\hline \multicolumn{4}{|l|}{ Age $(y r)$} \\
\hline Mean \pm SD & $46.6 \pm 7.9$ & $44.8 \pm 8.4$ & \multirow[t]{2}{*}{0.03} \\
\hline Range & $19-69$ & $18-70$ & \\
\hline \multicolumn{3}{|l|}{ Gender } & \multirow[t]{3}{*}{ ns } \\
\hline Male & $139(63 \%)$ & $119(64 \%)$ & \\
\hline Female & $81(37 \%)$ & $67(36 \%)$ & \\
\hline \multicolumn{3}{|l|}{ Race } & \multirow[t]{7}{*}{0.03} \\
\hline Caucasian & $179(80.9 \%)$ & $129(69.4 \%)$ & \\
\hline African-American & $20(9.1 \%)$ & $26(14 \%)$ & \\
\hline Native American & $10(4.5 \%)$ & & \\
\hline Hispanic & $5(2.3 \%)$ & & \\
\hline Asian & $1(0.5 \%)$ & & \\
\hline Unknown/other & $5(2.3 \%)$ & $31(16.6 \%)$ & \\
\hline \multicolumn{4}{|l|}{ Educational level } \\
\hline Some high school & $21(9.6 \%)$ & & \\
\hline High school graduate & $136(61.8 \%)$ & & \\
\hline College graduate & $63(28.6 \%)$ & & \\
\hline \multicolumn{4}{|l|}{ Marital status } \\
\hline Single, never married & $26(11.8 \%)$ & & \\
\hline Married & $144(65.5 \%)$ & & \\
\hline Divorced & $43(19.5 \%)$ & & \\
\hline Widowed & $4(1.8 \%)$ & & \\
\hline Separated & $3(1.4 \%)$ & & \\
\hline \multicolumn{4}{|l|}{ Employment } \\
\hline Working (full/part time) & $152(69 \%)$ & & \\
\hline Housewife or homemaker & $18(8.2 \%)$ & & \\
\hline Retired & $14(6.4 \%)$ & & \\
\hline Unemployed & $12(5.5 \%)$ & & \\
\hline Student & $4(1.8 \%)$ & & \\
\hline Other & $18(8.2 \%)$ & & \\
\hline \multicolumn{4}{|l|}{ Serum ALT } \\
\hline Mean \pm SD & $115 \pm 89 \mathrm{IU} / \mathrm{L}$ & & \\
\hline No. with normal values $(<46$ IU/L) & $39(20.5 \%)$ & & \\
\hline \multicolumn{4}{|l|}{ Cirrhosis } \\
\hline Yes & $40(34.8 \%)$ & & \\
\hline No & $75(65.2 \%)$ & & \\
\hline No biopsy data in last $2 \mathrm{yr}$ & $105(47.7 \%)$ & & \\
\hline \multicolumn{4}{|l|}{ Treatment } \\
\hline Treatment naive & $168(76.3 \%)$ & & \\
\hline No sustained response to previous $\mathrm{Rx}$ & $52(23.6 \%)$ & & \\
\hline \multicolumn{4}{|l|}{ Intravenous drug use } \\
\hline Yes & $111(50.4 \%)$ & & \\
\hline Drug dependence & $67(31 \%)$ & & \\
\hline Drug rehabilitation & $61(27.7 \%)$ & & \\
\hline No & $109(49.6 \%)$ & & \\
\hline \multicolumn{4}{|l|}{ Regular alcohol consumption } \\
\hline Yes & $119(54.1 \%)$ & & \\
\hline Alcohol dependence & $47(21.7 \%)$ & & \\
\hline Alcohol rehabilitation & $54(24.5 \%)$ & & \\
\hline No & $101(45.9 \%)$ & & \\
\hline
\end{tabular}

sian and 9\% African-American. Of the patients, $62 \%$ had completed high school and $29 \%$ were college graduates. When the study population was compared to eligible nonparticipants, there was no difference in gender; however, the study population was slightly older and more likely to be Caucasian (Table 1).

Serum ALT was within the normal range in 45 (20\%) patients. Of the $115(52 \%)$ patients who had liver biopsies within the last $2 \mathrm{yr}, 40(35 \%)$ had cirrhosis.

\section{Comorbid Illness}

One or more comorbid medical or psychiatric illnesses was present in 157 (71\%) patients (Table 2); 114 patients (51.8\%) had medical illness only, 36 (16.4\%) had both medical and psychiatric illnesses, and seven (3.2\%) had psychiatric illness only. The common medical and psychiatric diagnoses are listed in Table 2. Among the patients with medical comorbid illness only, 59 (52\%), 32 (28\%), and $23(20 \%)$ patients had 1,2 , and $\geq 3$ medical diagnoses, 
Table 2. List of Common Comorbid Illnesses

\begin{tabular}{lc}
\hline \multicolumn{1}{c}{ Illness } & No. of Patients $(\%)$ \\
\hline Medical diagnoses & \\
Osteoarthritis & $61(27.7)$ \\
Hypertension & $48(21.8)$ \\
Diabetes mellitus & $23(10.5)$ \\
Asthma & $19(8.6)$ \\
GERD & $17(7.7)$ \\
Hypothyroidism & $15(6.8)$ \\
Cardiovascular diseases & $16(7.3)$ \\
Other pulmonary diseases & $3(1.3)$ \\
Other musculoskeletal disorders & $27(12.3)$ \\
Other gastrointestinal diseases & $25(11.4)$ \\
Neurologic diseases & $14(3.6)$ \\
Renal diseases & $6(2.7)$ \\
Miscellaneous diseases & $13(5.9)$ \\
Psychiatric diagnoses & \\
Depression & $29(13.2)$ \\
Anxiety & $7(3.2)$ \\
Miscellaneous diagnoses & $7(3.2)$ \\
\hline
\end{tabular}

respectively. Of the patients with combined medical and psychiatric illnesses 12 (33\%), 10 (28\%), and 14 (39\%) patients had 1,2 , and $\geq 3$ medical diagnoses, respectively. The mean number of comorbid medical illness in these two groups was similar (1.8 vs 2.2/patient, ns).

\section{Substance Abuse}

A total of $111(50 \%)$ patients reported a history of i.v. drug use, $67(31 \%)$ considered themselves to be dependent on recreational drugs at some stage of their lives, and 61 (27.7\%) reported prior counseling or rehabilitation for drug abuse (Table 1). In addition, 119 (54\%) patients reported a history of regular alcohol consumption, 47 (22\%) considered themselves to have been dependent on alcohol at some stage of their lives, and 54 (24.5\%) reported prior counseling or rehabilitation for alcohol abuse (Table 1).

\section{HRQOL in CHC Patients}

The mean SF-36 scores in our CHC patients were significantly lower than those of the general U.S. as well as the healthy U.S. population in all eight subscales and the two summary scales $(p<0.001)$ (Fig. 1). Because there was a preponderance of male participants, comparison with the general U.S. population was repeated after controlling for gender. The SF-36 scores in our CHC patients remained significantly lower than age and gender matched general U.S. population $(p<0.001)$.

To determine whether HRQOL scores for our CHC patients were lower than for $\mathrm{CHC}$ patients selected for treatment trials, we compared the SF-36 scores of our patients with the pretreatment scores from the largest published study of HRQOL in CHC patients enrolled in a treatment trial (2). The SF-36 scores in our patients were lower in all eight subscales, the difference being significant in five subscales (PF, BP, GH, RE, and MH, $p<0.001$ ) (Fig. 2). Similar findings were observed when our results were com-

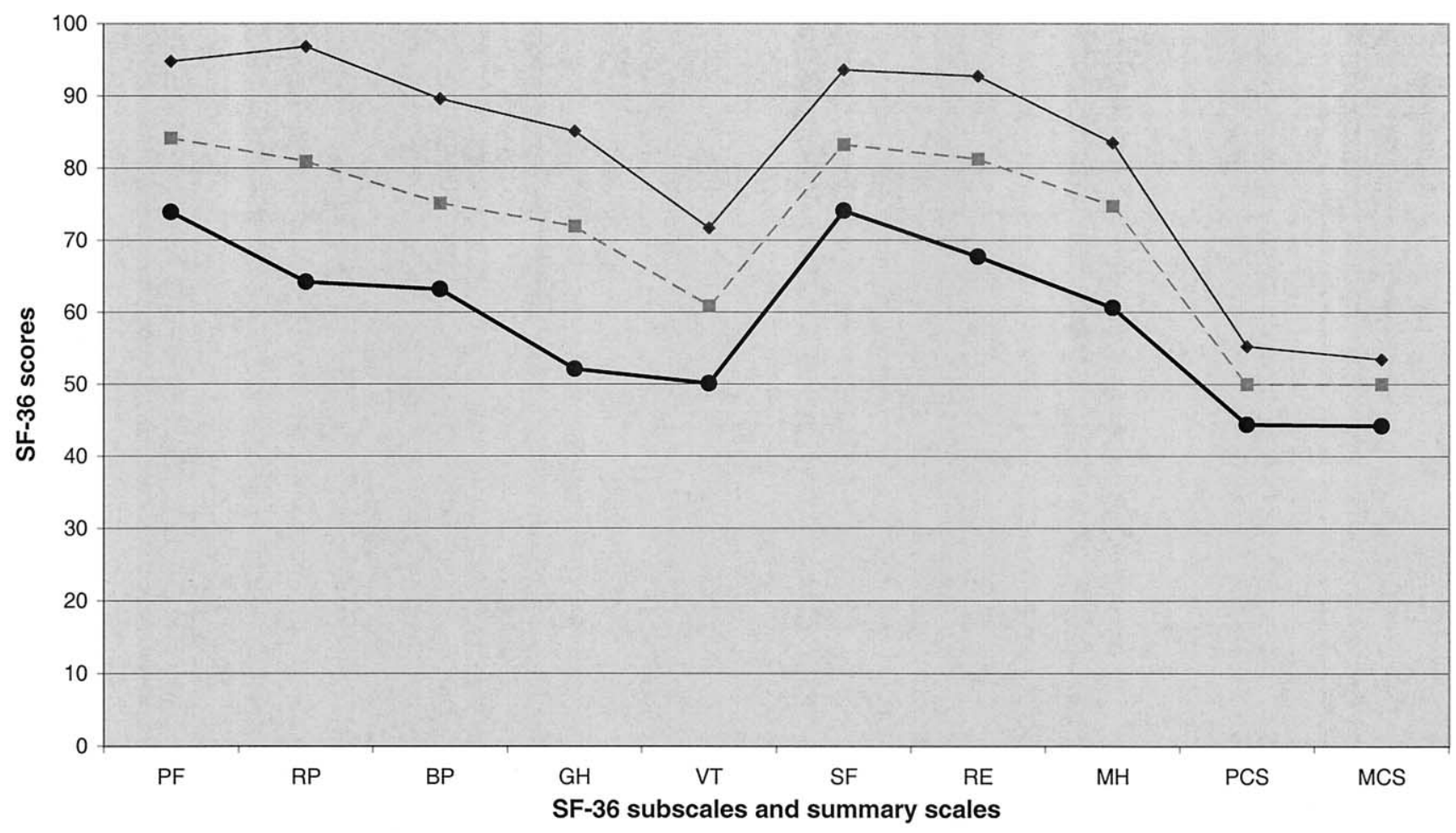

$\longrightarrow \mathrm{CHC}$ group $(\mathrm{N}=220)--\mathrm{n}-$ "General" U.S. pop $(\mathrm{N}=2474) \longrightarrow$ "Healthy" U.S. pop (N=465)

Figure 1. Health-related quality of life in chronic hepatitis $C$ patients versus healthy and general U.S. population. 


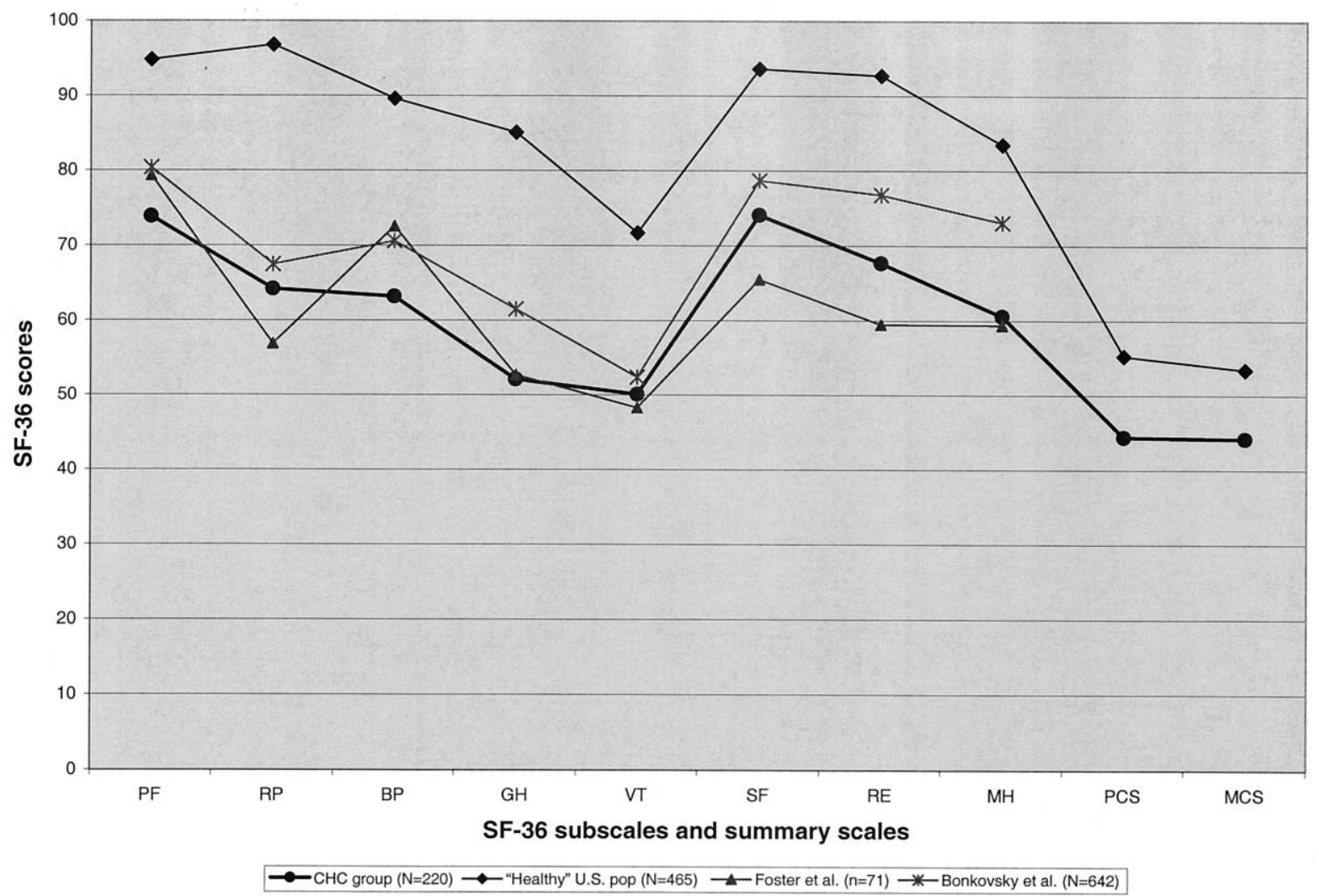

Figure 2. Health-related quality of life in chronic hepatitis C patients versus healthy U.S. population and CHC patients in two published reports.

pared to the pretreatment scores of CHC patients participating in a retreatment trial for relapsers (data not shown) (6).

To determine whether HRQOL scores for our CHC patients were lower than for other CHC patients who were not participating in treatment trials, we compared the SF-36 scores of our patients with those reported by Foster et al. (3). The SF-36 scores in our patients were lower in two subscales (PF, $p=0.004$; BP, $p=0.001$ ), higher in two subscales (SF, $p<0.001 ; \mathrm{RE}, p=0.003$ ) and similar in the remaining four subscales (Fig. 2).

\section{HRQOL and Comorbid Illness}

Compared to the healthy US population, the $63 \mathrm{CHC}$ patients with no comorbid medical or psychiatric illness had significantly lower SF-36 scores in all subscales and summary scales ( $p \leq 0.003$ ) (Fig. 3). The SF-36 scores were more markedly reduced among the $157 \mathrm{CHC}$ patients with comorbid illness $(p<0.001)$.

Among the patients with comorbid illness, HRQOL was more profoundly affected in patients who had a psychiatric diagnosis. Compared to $\mathrm{CHC}$ patients with no comorbid illness, the presence of a psychiatric diagnosis alone resulted in significant reductions in SF-36 scores in four subscales and one summary scale, whereas the presence of a combination of psychiatric and medical illnesses resulted in significant reductions in SF 36-scores in all subscales and both summary scales (Fig. 3). Comorbid medical illness alone had less impact on the SF-36 scores in patients with CHC, with no appreciable change in SF-36 scores in patients with one comorbid medical illness, as well as significant reductions in SF-36 scores in only two subscales and the physical summary scale in patients with two comorbid medical illnesses. Patients with three or more comorbid medical illnesses had greater reductions in HRQOL, with significant reduction in SF-36 scores in six subscales and the physical summary scale compared to $\mathrm{CHC}$ patients with no comorbid illness.

\section{HRQOL and Demographic Factors}

There was no correlation between age and race with SF-36 scores in our study population. Women tended to have lower SF-36 scores compared to men, but a significant difference was noted only in selected physical subscales (Table 3). Similarly, level of education and marital status demonstrated weak correlations with a few SF-36 subscales in the expected direction (i.e., individuals with a higher level of education and married individuals had higher SF-36 scores) (Table 3).

\section{HRQOL and Liver Disease Parameters}

There was no significant correlation between any SF-36 subscales or summary scores and the liver disease parameters of serum ALT level or the presence of cirrhosis on liver biopsy. CHC patients who were nonresponders to previous 


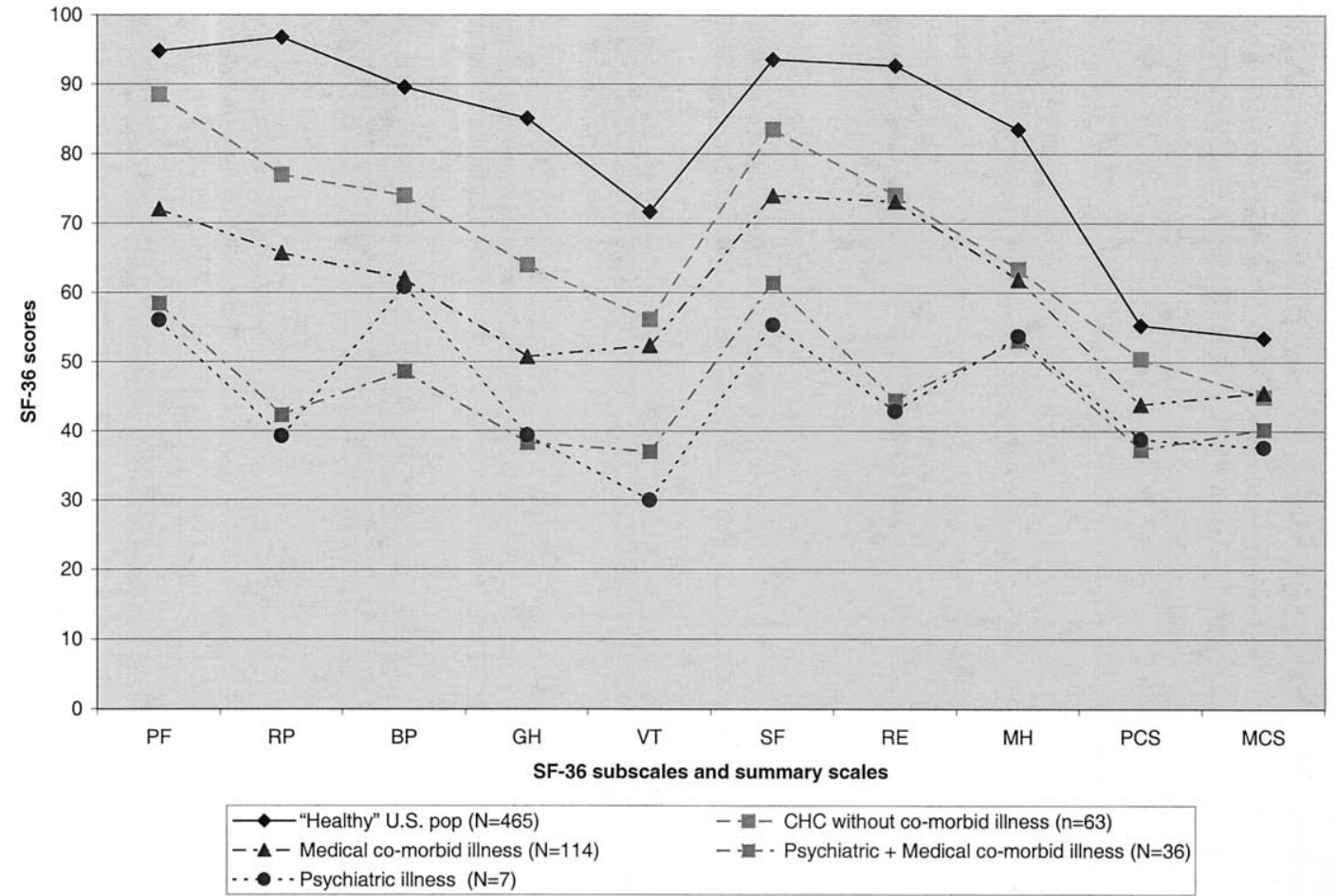

Figure 3. Health-related quality of life in chronic hepatitis $\mathrm{C}$ patients with no comorbid medical illness versus $\mathrm{CHC}$ patients with comorbid medical or psychiatric illness, and healthy U.S. population.

treatment had SF-36 scores similar to those of treatmentnaive patients.

\section{HRQOL and History of Substance Abuse}

There was no significant correlation between SF-36 subscale or summary scores and history of drug or alcohol use or dependence.

\section{DISCUSSION}

Similar to other published reports, we found that the HRQOL in our CHC patients was significantly impaired when compared to U.S. healthy and general populations. Our study also showed that the HRQOL scores in unselected $\mathrm{CHC}$ patients were significantly lower than $\mathrm{CHC}$ patients enrolled in treatment trials (2). These findings suggest that previous studies may have underestimated the extent of impairment in HRQOL in CHC patients. We acknowledge that our study was performed in a different clinical setting and that the tools we used to assess HRQOL were not identical to that in the study by Bonkovsky et al. (2). In addition, comparisons were made based on overall results of the two groups of patients and not on actual data. These methodological problems may bias our conclusions. Nevertheless, both studies used SF-36 as the core instrument to assess HRQOL. Moreover, comparisons of the actual data in this study with those of our previous study of 107 patients enrolled in a retreatment trial (11) also showed that patients in the current study had significantly lower subscale and summary scores. The SF-36 scores in our patients were comparable to those reported in another, unselected $\mathrm{CHC}$ patient population (3), lending support to our hypothesis that HRQOL in the general pool of $\mathrm{CHC}$ patients is lower than in CHC patients who qualify for entry into clinical trials.

We acknowledge that our results may not be generalized to all CHC patients because of the enrollment rate of $54 \%$ and selection of patients referred to a liver clinic. The design of our study required an extra $45 \mathrm{~min}$ in addition to the clinic visit. Although time constraint was the major reason for

Table 3. Univariate Analysis Comparing HRQOL and Demographic Characteristics

\begin{tabular}{lccccccccccc}
\hline \multicolumn{10}{c}{ SF-36 Subscales and Summary Scales } & & \\
\hline & PF & RP & BP & GH & VT & SF & RE & MH & PCS & MCS \\
\hline Age & $\mathrm{ns}$ & $\mathrm{ns}$ & $\mathrm{ns}$ & $\mathrm{ns}$ & $\mathrm{ns}$ & $\mathrm{ns}$ & $\mathrm{ns}$ & $\mathrm{ns}$ & $\mathrm{ns}$ & 0.006 \\
Race & $\mathrm{ns}$ & $\mathrm{ns}$ & $\mathrm{ns}$ & $\mathrm{ns}$ & $\mathrm{ns}$ & $\mathrm{ns}$ & $\mathrm{ns}$ & $\mathrm{ns}$ & $\mathrm{ns}$ & $\mathrm{ns}$ \\
Gender & 0.001 & $\mathrm{~ns}$ & 0.006 & 0.001 & 0.001 & 0.005 & $\mathrm{~ns}$ & $\mathrm{~ns}$ & 0.001 & $\mathrm{~ns}$ \\
Education & 0.006 & $\mathrm{~ns}$ & $<.001$ & 0.01 & $\mathrm{~ns}$ & 0.009 & $\mathrm{~ns}$ & $\mathrm{~ns}$ & 0.001 & $\mathrm{~ns}$ \\
Marital status & 0.005 & $\mathrm{~ns}$ & 0.003 & 0.008 & $\mathrm{~ns}$ & $<.001$ & $\mathrm{~ns}$ & 0.006 & 0.006 & $\mathrm{~ns}$ \\
\hline
\end{tabular}


study refusal, it was our belief that data provided by the patient in a private environment with no influence from family or friends are more reliable than those from a questionnaire completed and mailed from home. Our study design also allowed us to have a more accurate and complete assessment of comorbid illness. Compared to the eligible CHC patients who did not participate in this study, our patients were similar in gender, with a difference in mean age of only $1.8 \mathrm{yr}$. The higher proportion of Caucasians in our study compared to the patients who declined to participate may have created a small bias in our results. However, our data showed that there was no correlation between HRQOL and race or age of the participants.

As in our previous study of patients with IFN refractory CHC (11), we found that the presence of comorbid illness was the most important predictor of HRQOL in CHC patients. However, CHC patients without comorbid illness also had significant impairment in SF-36 scores when compared to healthy individuals, indicating that $\mathrm{CHC}$ alone is sufficient to cause reduction in HRQOL. Our study demonstrated that comorbid illness in CHC patients led to further reduction in HRQOL. Moreover, a psychiatric diagnosis alone was associated with more marked decrease in SF-36 scores compared to those of medical illness. The impact of comorbid illness, in particular psychiatric illness, on HRQOL in CHC patients may account for the lower SF-36 scores in our patients compared to those selected for treatment trials as patients with psychiatric illness or multiple comorbid medical illness are usually excluded from treatment trials. We acknowledge that the impact of comorbid psychiatric illness alone on HRQOL in CHC patients should be further studied, as there were only seven patients in this group.

In this study, women tended to have lower SF-36 scores, a finding that has been consistently reported even among healthy subjects (12) and in those with other chronic diseases (14). We also found weak associations between HRQOL and level of education as well as marital status. These associations are probably nonspecific and may reflect better socioeconomic status among patients with higher levels of education and less emotional distress among those who are married.

Contrary to our hypothesis, a history of drug use or dependence was not associated with a more marked diminution in HRQOL in our CHC patients. This is different from the study by Foster et al., which found that patients with a history of i.v. drug use had lower SF-36 scores. This finding was unexpected, as previous studies have shown that subjects with a history of i.v. drug use have psychological disturbances that may lead to a reduction in HRQOL (15, 16). The reason for the lack of association in our study may be related to the fact that the history of i.v. drug use was remote in most patients. We also found no association between a history of alcohol use or dependence and HRQOL in our CHC patients.

As in other studies $(2-7,10)$, the liver disease parameters of serum ALT or presence of cirrhosis on liver biopsy did not correlate with SF-36 scores in our CHC patients. We acknowledge that only half of our patients had liver biopsies in the last $2 \mathrm{yr}$, so the impact of compensated cirrhosis on HRQOL in CHC patients cannot be accurately assessed. Other studies showed that viral factors were also not associated with SF-36 scores in CHC patients. Thus, the reasons for diminished HRQOL in CHC patients with no comorbid illness remain unclear.

In summary, we have clearly demonstrated that HRQOL is significantly impaired in CHC patients, and that the reduction in HRQOL is more marked in unselected $\mathrm{CHC}$ patients compared to CHC patients who are selected for entry into treatment trials. The presence of comorbid illness is the most important predictor of HRQOL in CHC patients, but $\mathrm{CHC}$ alone is sufficient to result in significant diminution in HRQOL compared to that of healthy individuals. These findings underscore the health burden of $\mathrm{CHC}$ even in ostensibly asymptomatic patients, and highlight the need to develop more effective and better-tolerated treatments that can benefit more patients with CHC. Our results also emphasize the importance of managing other comorbid illness in CHC patients, as well as the need to conduct research into the mechanisms of impaired HRQOL among patients with CHC alone.

\section{ACKNOWLEDGMENTS}

We thank Ms Karen Boase, Diane Peyton, Amy RandallRay, and Pamela Richtmyer for their assistance with this study, and the hepatology faculty at University of Michigan for their assistance with patient recruitment. This study was supported in part by an unrestricted grant from Schering Plough Corporation and National Institutes of Health MO1RR00042 to the General Clinical Research Center at the University of Michigan. Dr. Hussain was supported by a Schering Plough Hepatology Fellowship.

Reprint requests and correspondence: Anna S. F. Lok, M.D., Division of Gastroenterology, University of Michigan Hospitals, 3912 Taubman Center, Box 0362, Ann Arbor, MI 48109.

Received Jan. 12, 2001; accepted Apr. 26, 2001.

\section{REFERENCES}

1. Alter MJ, Kruszon-Moran D, Nainan OV, et al. The prevalence of hepatitis C virus infection in the United States, 1988 through 1994. N Engl J Med 1999;341:556-62.

2. Bonkovsky HL, Woolley JM, Consensus Interferon Study Group. Reduction of health-related quality of life in chronic hepatitis $\mathrm{C}$ and improvement with interferon therapy. Hepatology 1999;29:264-70.

3. Foster GR, Goldin RD, Thomas HC. Chronic hepatitis C virus infection causes significant reduction in quality of life in the absence of cirrhosis. Hepatology 1998;27:209-12.

4. Hunt CM, Dominitz, JA, Bute BP, et al. Effect of interferon treatment of chronic hepatitis $\mathrm{C}$ on health-related quality of life. Dig Dis Sci 1997;42:2482-6. 
5. Bayliss MS, Gandek B, Bungay KM, et al. A questionnaire to assess generic and disease specific health outcomes of patients with chronic hepatitis C. Qual Life Res 1998;7:39-55.

6. Ware JE, Bayliss MS, Mannocchia M, et al. Health-related quality of life in chronic hepatitis C. Impact of disease and treatment response. Hepatology 1999;30:550-5.

7. Davis GL, Balart LA, Schiff ER, et al. Assessing healthrelated quality of life in chronic hepatitis $\mathrm{C}$ using the Sickness Impact Profile. Clin Ther 1994;16:334-43.

8. Tarlov AR, Ware JE, Greenfield S, et al. Medical outcomes study. An application of methods for evaluating the results of medical care. JAMA 1989;262:925-30.

9. Ware JE, Sherbourne CD. The MOS 36 Item Short Form Health Survey (SF 36): Conceptual framework and item selection. Med Care 1992;30:473-83.

10. Carithers RL, Sugano D, Baylis MS. Health assessment for chronic HCV infection, results of quality of life. Dig Dis Sci 1996;41:75-80S.
11. Fontana RJ, Moyer CA, Sonnad S, et al. Comorbidities and quality of life in patients with interferon-refractory chronic hepatitis C. Am J Gastroenterol 2001;96:170-8.

12. Ware JE, Snow KK, Kosinkio M, et al. SF-36 Health Survey manual, and interpretation guide. Boston: New England Medical Center, 1993.

13. Ware JE, Kosinski M, Keller SD. SF-36 Physical and Mental Health Summary scales: A user's manual. Boston: New England Medical Center, 1994

14. Osborne ML, Vollmer WM, Linton KL, et al. Characteristics of patients with asthma within a large HMO: A comparison by age and gender. Am J Resp Crit Care Med 1998;157:123-8.

15. Kendall J, Sherman M, Bigelow G. Psychiatric symptoms in polysubstance abusers: Relationship to race, age, sex and age. Addict Behav 1995;20:685-90.

16. Dinwiddie SH, Reich T, Clininger RC. Psychiatric comorbidity and suicidality among intravenous drug abusers. J Clin Psych 1992;53:364-9. 